\title{
Predictors of Nerve Conduction Study Parameters among Type 2 Diabetic Patients: A Cross -Sectional Study
}

\author{
Wakode $\mathrm{SL}^{* 1}$, Thakre $\mathrm{AE}^{1}$, Hulke $\mathrm{SM}^{1}$ and Wakode $\mathrm{NS}^{2}$ \\ ${ }^{1}$ Associate Professor, Department of Physiology, AIIMS BHOPAL, India \\ ${ }^{2}$ Associate Professor, Department of Anatomy Government Medical College Vidisha, India
}

Received: 製: November 08, 2018; Published: 製: November 19, 2018

*Corresponding author: Santosh Laxman Wakode, Associate Professor, Department of Physiology, AIIMS Bhopal, India

\begin{abstract}
The prevalence of diabetes is rapidly rising all over the globe and India is becoming the world capital for diabetic patients. Persistent hyperglycemia leads to micro vascular complications like diabetic peripheral neuropathy (DPN) which contributes to significant morbidity in the society. Recent research reported that NCS could be used for the early detection \& prediction of DPN.The present cross -sectional study was carried to explore relations of NCS parameters and different variables among type 2 diabetic patients. Data of total 50 (male \& female) type 2 diabetic patients on regular oral hypoglycemic agents with disease duration not less than two years was collected and analyzed to find out correlations between NCS parameters like motor \& sensory conduction velocity (MNCV, SNCV), motor and sensory action potential amplitude (CMAP, SNAP) with duration of disease, fasting \& post meal blood glucose (FBG, PMBG) \& body mass index (BMI). Significant negative correlation (p<0.05) was seen between the disease duration and all the NCS parameters studied, between the fasting blood glucose (mean value $137.88 \pm 34.14 \mathrm{mg} / \mathrm{dl}$ ) and MNCV and SNAP, between post meal blood glucose (mean value $213.90 \pm 67.72 \mathrm{mg} / \mathrm{dl}$ ) and MNCV, SNCV, SNAP and also between BMI (mean value $28.94 \pm 4.27 \mathrm{~kg} / \mathrm{m} 2$ ) and MNCV and SNCV. The negative correlations of nerve conduction parameters with different variables indicates subclinical nerve dysfunction, therefore thorough neurological examination along with NCS should be carried out early in the course of disease.
\end{abstract}

Abbreviations: DPN: Diabetic Peripheral Neuropathy, MNCV: Motor Conduction Velocity, SNCV: Sensory Conduction Velocity, FBG: Fasting Blood Glucose, PMBG: Post Meal Blood Glucose, BMI: Body Mass Index, MNAP and SNAP: Motor and Sensory Action Potential Amplitude, NCS: Nerve Conduction Studies, DPN: Diabetic Polyneuropathy, DM: Diabetes Mellitus

\section{Introduction}

The prevalence of diabetes is rapidly rising all over the globe at an alarming rate and it is reaching potentially epidemic proportions in India. It has been predicted that global prevalence of diabetes will be doubled from 171 million in 2000 to 366 million by the year 2030, with maximum increase in Indian population [1]. It is a group of metabolic disorders characterized by hyperglycemia resulting from defect in insulin secretion, its action or both. Persistent hyperglycemia seen in diabetes mellitus (DM) by various mechanisms leads to potential macro \& micro vascular complications that pose significant health care burden to society and contribute significantly to morbidity \& mortality. Diabetic peripheral neuropathy (DPN) is one of the most common micro vascular complications of diabetes, however worsening of nerve function attributable to DM can be demonstrated before criteria for diabetic polyneuropathy are met [2].

Along with hyperglycaemia different variables like body mass index (BMI), disease duration, ethnicity, age, gender can influence neuropathic endpoints [3]. Recent research reported that nerve conduction studies (NCS) could be used for the early detection \& prediction of DPN $[4,5]$. However Various studies in the past reported variable electrophysiological abnormalities, also ethnic differences for NCS parameters among diabetic patients [6], further the correlations between different variables were not uniform [7-9]. There is scarcity in literature regarding research for early detection \& predictive roles of NCS for nerve damage in type 2 diabetic patients. So, the present cross-sectional study was carried out to explore relations between disease duration, BMI, fasting \& post meal blood glucose with nerve conduction study parameters among diabetes mellitus patients from central India. 


\section{Material and Methods}

The present cross-sectional study was carried out at one of the tertiary health care center of central India. Study subjects were recruited from the patients regularly attending diabetology clinic. On world diabetes day, to promote awareness about diabetes in society, free health check-up, motivational lectures and guidance camp was organized, which also included estimation of fasting \& post meal blood glucose (FBG, PMBG) and nerve conduction studies (NCS). Prior information about the event was given to the patient attending the diabetic OPD. The known diabetic patients who were willing to participate in health check-up camp were registered and schedule of the event was explained. There general demographic parameters were recorded and written informed consent was taken. On the world diabetes day, they were asked to come in the morning, overnight fasted to provide blood sample for blood sugar level. The study subjects were having freedom to withdraw any point of time during the whole schedule. Those participants who turned up in the morning and provided the blood sample went through the various events of the camp, had breakfast and again the blood sample was collected for post meal sugar estimation.

Those participants without any overt sign suggestive of peripheral neuropathy on clinical examination and given consent were selected for nerve conduction study. Right median nerve motor \& sensory nerve conduction study was carried out using standard protocol [10], upper limb skin temperature was maintained at $31^{\circ} \mathrm{C}$. Right median nerve motor nerve conduction velocity (MNCV), compound motor action potential (CMAP), antidromic sensory nerve conduction velocity (SNCS) \& sensory nerve action potentials (SNAP) were measured. In the last part of event printed reports of their biochemical parameters \& NCS were given and participants had freedom to discuss the report with any of the consultants. After getting hospital ethics committee clearance, demographic, biochemical \& NCS data of participants was used for analysis. Although total 200 patients had registered for the camp only 90 participants turned up for the event \& 60 completed nerve conduction study. Out of the total 90 participants the data of 50 middle age (male \& female) patients was selected for the analysis. The inclusion criterion for the data selection was:

a) Diabetics in the age range of 35-45 years,

b) Having disease duration of more than two years,

c) Regularly taking oral hypo glycaemic drug therapy.

The exclusion criterion for data selection was:

a) Patients on insulin therapy,

b) Disease duration less than 2 years,

c) Patients not maintaining overnight fasting,

d) Over aged participants and those with any other major systemic illness or peripheral neuropathy.

Blood glucose was determined by using glucose oxidase method with routine biochemistry analyzer. NCS was carried out using computerized RMS EMG EP MK II machine.

\section{Statistical Analysis}

Data compiled was checked for correctness and completeness. Body mass index was determined using standard formula (weight/ height2). Correlations between disease duration, fasting blood glucose, post meal blood glucose and BMI, with nerve conduction study parameters were done using Pearson's correlation coefficient, $r$ value was determined \& p value less than 0.05 was taken as level of significance.

\section{Results}

Data of total 50 participants was compiled and analyzed, all data was expressed as mean \& standard deviation. The ages ranged between 36 and 40 years, duration of DM ranged between 2 to 6 years. Table 1 gives details of the general, biochemical and nerve conduction study parameters of participants. Correlation between nerve conduction study parameters with disease duration, fasting blood glucose, post meal blood glucose \& body mass index is shown in Figure 1. Significant negative correlation $(p<0.05)$ is seen between the disease duration and all the NCS parameters studied, between the fasting blood glucose (mean value $137.88 \pm 34.14 \mathrm{mg} /$ $\mathrm{dl}$ ) and MNCV and SNAP, between post meal blood glucose (mean value $213.90 \pm 67.72 \mathrm{mg} / \mathrm{dl}$ ) and MNCV, SNCV, SNAP and also between BMI (mean value $28.94 \pm 4.27 \mathrm{~kg} / \mathrm{m}^{2}$ ) and MNCV and SNCV.

Table 1: General, biochemical \& nerve conduction study findings of study participants.

\begin{tabular}{|c|c|c|}
\hline Variable & Mean & Standard Deviation \\
\hline Age (years) & 38.58 & 2.58 \\
\hline Duration (years) & 4.18 & 2.61 \\
\hline BMI $\left(\mathrm{kg} / \mathrm{m}^{2}\right)$ & 28.94 & 4.27 \\
\hline FBG $(\mathrm{mg} / \mathrm{dl})$ & 137.88 & 34.14 \\
\hline PMBG $(\mathrm{mg} / \mathrm{dl})$ & 213.90 & 67.72 \\
\hline MNCV $(\mathrm{m} / \mathrm{s})$ & 52.56 & 8.58 \\
\hline SNCV $(\mathrm{m} / \mathrm{s})$ & 46.77 & 10.04 \\
\hline $\mathrm{CMAP}(\mathrm{mv})$ & 4.92 & 2.16 \\
\hline SNAP $(\mu \mathrm{v})$ & 13.09 & 4.99 \\
\hline
\end{tabular}

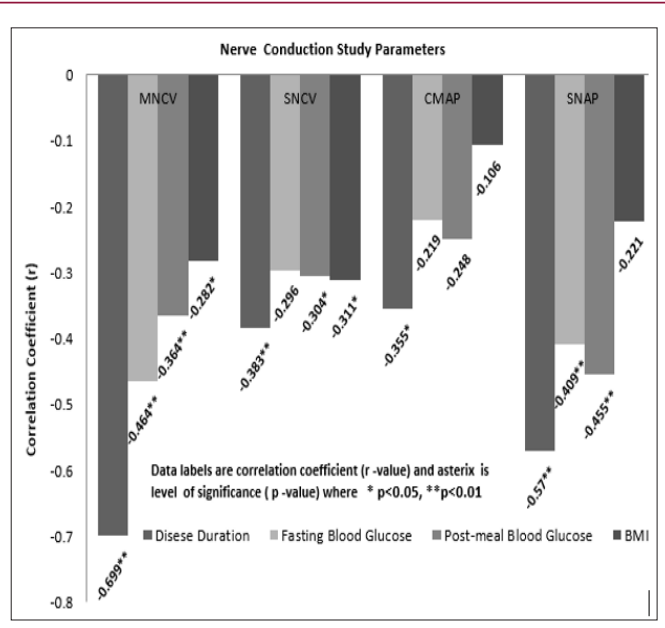

Figure 1: Correlation between nerve conduction study parameters and Disease duration, Fasting blood glucose, post meal blood glucose and BMI. 


\section{Discussion}

The present cross-sectional study was carried out among type 2 diabetes mellitus patients who were without any overt clinical signs suggestive of peripheral neuropathy, and correlations of different nerve conduction parameters with disease duration, FBG, PMBG \& BMI was examined. MNCV was significantly negatively correlated with all the variables studied. SNCV was significantly negatively correlated with all the variables studied except fasting blood glucose. CMAP was significantly negatively correlated only with disease duration, other variables studied have shown the moderate strength negative correlation, but it was not statistically significant. SNAP was significantly negatively correlated with all the variables studied except BMI. Thus, it indicates association between worsening of nerve function \& diabetes mellitus before criteria for diabetic polyneuropathy is met. These findings are attributable to various risk factors associated with DM like hyperglycaemia, BMI \& disease duration [2,11]. Abnormalities in NCS parameters, which are frequently detected in subclinical diabetic neuropathy conditions, are the first objective indicator of diabetic peripheral neuropathy. NCS evaluates only large myelinated fibres of peripheral nerves and it reflects primarily three conditions normal, demyelination, or axonal loss [5]. Normal means myelin \& axons are intact which shows normal amplitude \& conduction velocity, axon loss reflect in reduced amplitude \& loss of myelin shows reduced conduction velocity during NCS evaluation [10].

In agreement with the findings of previous workers $[4,12]$ the present study showed significant negative correlations of NCS parameters without clinically evident peripheral neuropathy, these subclinical reductions of nerve conduction study parameters can predict manifestation of clinical peripheral neuropathy few years later. In the present study, motor \& sensory conduction velocity showed significant negative correlation with almost all variables, thus the predominant pattern of involvement had a demyelinating trend, but not enough to fulfil the criteria for demyelinating neuropathy. Sensory amplitudes showed significant negative correlations with duration of disease \& glycaemic control, while motor amplitudes were negatively correlated with duration of disease, which suggest axonal involvement at the same time with demyelination. This indicates that among diabetic patients, alteration of peripheral nerve parameters begins at the early stage of disease and its further progression to peripheral neuropathy is attributable to various risk factors [13].

Mean FBG \& PMBG values observed in current study were suggestive of poorglycaemic control \& was negatively correlated with conduction velocity. These raised blood glucose level in diabetics can decrease nitric oxide in nerves causing endoneural hypoxia resulting in slowed conduction [14-16]. However mild reversible reduction in conduction velocity can be seen without structural changes in myelin due to metabolic causes like hyperglycaemias [5]. Inflammation, oxidative stress \& mitochondrial dysfunction are the three main alterations responsible for peripheral nerve dysfunction, whereas, duration, uncontrolled hyperglycaemia \& BMI are the risk factors frequently associated with it. Decreased nitric oxide can generate reactive oxygen species causing inflammation which facilitates production of inflammatory cytokines that leads to nerve damage and this trend further continues with disease duration $[17,18]$. Increased BMI and obesity lead to insulin resistance and worsens hyperglycaemia that can further augment diabetic complications $[15,19]$. Researchers in the past also depicted ethnic influences on severity of DPN, with black non-Hispanic, mixed, or Asian patients more affected than Caucasians [6].

The main limitations of the present study are that, the data obtained was cross-sectional with small sample size and although the analysis suggests an association between NCS \& different variables these conclusions are rather speculative, also the possibility of subclinical carpal tunnel syndrome was not ruled out during recording of median nerve conduction study. Other limitation is, only upper limb nerve conduction parameters and limited variables were studied for correlations. Therefore, further long-term longitudinal study with large sample size is required. The result of the current study came out with negative correlations between NCS parameters \& different variables in diabetic patients, therefore we recommend that for early detection \& prediction of DPN, thorough neurological examination for neurological signs \& symptoms with good clinical proficiency should be carried out early in the course of disease and NCS findings could be used accordingly.

\section{References}

1. Kaveeshwar SA, Cornwall J (2014) The current state of diabetes mellitus in India. Australas Med J 7(1): 45-48.

2. Dyck PJ, O brien PC, Litchy WJ (2005) Monotonicity of nerve tests in diabetes. Subclinical nerve dysfunction precedes diagnosis of polyneuropathy. Diabetes Care 28(9): 2192-2200.

3. Dyck PJ, Litchy WJ, Lehman KA, Hokanson JL, Low PA (1995) Variables influencing neuropathic endpoints: the Rochester Diabetic Neuropathy Study of Healthy Subjects. Neurology 45(6): 1115-11221.

4. Hyllienmark L, Alstrand N, Jonsson B (2013) Early electrophysiological abnormalities and clinical neuropathy: a prospective study in patients with type 1 diabetes. Diabetes Care 36(10): 3187-3194.

5. Jin HY, Park TS (2015) Can nerve conduction studies detect earlier and predict clinical diabetic neuropathy? J Diabetes Investig 6(1): 18-20.

6. Pop-Busui R, Lu J, Lopes N, Jones TLZ (2009) Prevalence of diabetic peripheral neuropathy and relation to glycemic control therapies at baseline in the BARI 2D cohort. Journal of the Peripheral Nervous System 14(1): 1-13.

7. Partanen J, Niskanen L, Lehtinen J, Mervaala E, Siitonen O, et al. (1995) Natural history of peripheral neuropathy in patients with non-insulin-dependent diabetes mellitus. N Engl J Med 333(2): 89-94.

8. Lehtinen JM, Uusitupa M, Siitonen O, Pyörälä K (1989) Prevalence of neuropathy in newly diagnosed NIDDM and nondiabetic control subjects. Diabetes 38(10): 1307-1313.

9. Singh RB, Chandel K, Kumar S (2015) Nerve conduction study findings of subclinical diabetic neuropathy in newly diagnosed diabetic patients. Indian J Neurosci 1: 1-7.

10. Wakode S, Barmate N, Ramteke S (2013) Basic Principles \& interpretation of Nerve Conduction Study : a Short Review. International Journal of Physiology 1(1): 26-31.

11. EL Saleem K, Ammari F, Khader Y, Dhaimat O (2009) Elevated glycosylated hemoglobin is associated with subclinical neuropathy in neurologically asymptomatic: A prospective study. J Clin Neurophysiol 26(1): 50-53. 
12. Weisman A, Bril V, Ngo M (2013) Identification and prediction of diabetic sensorimotor polyneuropathy using individual and simple combinations of nerve conduction study parameters. PLoS 8(3): e58783.

13. Román Pintos LM, Villegas Rivera G, Rodríguez Carrizalez AD, Miranda Díaz AG, Cardona Muñoz EG (2016) Diabetic Polyneuropathy in Type 2 Diabetes Mellitus: Inflammation, Oxidative Stress, and Mitochondrial Function. Journal of diabetes research.

14. Negi G, Kumar A, Joshi RP, Ruby PK, Sharma SS (2011) Oxidative stress and diabetic neuropathy: current status of Antioxidants. Institute of Integrative Omics and Applied Biotechnology Journal 2 (6): 71-78.

15. Knuiman MW, Welborn TA, McCann VJ, Stanton KG, Constable IJ (1986) Prevalence of diabetic complications in relation to risk factors. Diabetes 35 (12): 1332-1339.

ISSN: 2574-1241

DOI: 10.26717/BJSTR.2018.11.002060

Santosh Laxman Wakode. Biomed J Sci \& Tech Res

(C) (P) This work is licensed under Creative

Submission Link: https://biomedres.us/submit-manuscript.php
16. Akaza M, Akaza I, Kanouchi T, Sasano T, Sumi Y (2018) Nerve conduction study of the association between glycemic variability and diabetes neuropathy. Diabetology \& metabolic syndrome 10: 69.

17. Liu GH, Qu J, Shen X (2008) NF- $\kappa$ B/p65 antagonizes Nrf2- ARE pathway by depriving CBP from Nrf2 and facilitating recruitment of HDAC3 to MafK. Biochimica et Biophysica Acta-Molecular Cell Research 1783( 5): 713-727.

18. Ganesh Yerra V, Negi G, Sharma SS, Kumar A (2013) Potential therapeutic effects of the simultaneous targeting of theNrf2 and NF- $\kappa$ B pathways in diabetic neuropathy. Redox Biology (1)1: 394-397.

19. Inoue R, Sumitani M, Yasuda T (2016) Independent risk factors for positive and negative symptoms in patients with diabetic polyneuropathy. Journal of Pain \& Palliative Care Pharmacotherapy 30(3): 178-183.

$\begin{array}{ll}\text { BIOMEDICAL } & \text { Assets of Publishing with us } \\ \text { RESEARCHES } & \text { - Global archiving of articles } \\ \text { - Immediate, unrestricted online access } \\ \text { - Rigorous Peer Review Process }\end{array}$

